\title{
DEMONSTRATION OF THE HUMAN HAIR FOLLICLE MAGNETORECEPTION OF BIOMAGNETISM RADIATED BY THE CONCAVE PART OF THE HUMAN HAND
}

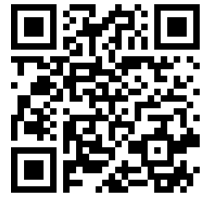

\author{
Abrahám A. Embí ${ }^{*}$ 凶 \\ ${ }^{*}$ BS, MBA, 13442 SW 102 Lane Miami Florida, USA 33186
}

DOI: https://doi.org/10.29121/granthaalayah.v8.i5.2020.291

Article Type: Research Article

Article Citation: Abrahám A. Embí. (2020). DEMONSTRATION OF THE HUMAN HAIR FOLLICLE MAGNETORECEPTION OF BIOMAGNETISM RADIATED BY THE CONCAVE PART OF THE HUMAN HAND. International Journal of Research -GRANTHAALAYAH, 8(5), $348-354$.

https://doi.org/10.29121/granthaa layah.v8.i5.2020.291

Received Date: 15 May 2020

Accepted Date: 31 May 2020

Keywords:

Hand Palm Biomagnetism Human Hair Magnetoreception Intrabody Energy Transfer Body parts Energy Exchange Potassium Ferrocyanide

\section{ABSTRACT}

Biological material has been documented to produce an external magnetic field that radiates out. There have been several papers documenting the magnetic fields produced by steady currents in the body. The most notable was published in 1980 by Cohen et al. where the human hair follicle was used as sentinel and biophysically evaluated via sophisticated equipment such as a double planar Superconducting Quantum Interference Devices (SQUID). Most recently, in 2019 Cohen's work was duplicated by Khan,S by also using double-planar gladiometers. Of interest to this manuscript is that since the introduction of anovel optical microscopy method in 2016 by Scherlag BJ et al is that numerous papers have been introduced in the literature now identifying intrinsic biomagnetic properties of the follicle such as penetration through glass barriers. In this manuscript, a concept of biomagnetic fields by the concave part of the human hand transferring energy to hair follicles is introduced, this was accomplished by using a novel optical microscopy method, in other words, the hair follicle is not limited to radiate out biomagnetism; but also, to receive externally radiated biomagnetic fields from a body part. This magneto receptive property is herein introduced.

\section{INTRODUCTION}

The purpose of this manuscript is to introduce evidence of radiated biomagnetic fields (BMFs) from the concave part of the human hand onto freshly plucked in toto scalp hairs (follicles and shaft) placed on a glass slide. Prior research done 40 years ago, demonstrated the prevalence magnetic fields produced by steady currents in the human body; the hair follicles over the head were identified as a reliable source producing reproducible patterns [1],[2]. Recently, with the introduction of an optical microscopy tabletop method [3], inherent electromagnetic energy emanating from isolated human hairs was also documented [4]. Until now, in the human hair follicle and shaft only forward moving source of BMFs emissions have been demonstrated, such as penetrating glass barriers [5]. This manuscript introduces a new finding, which is, which is documentation of air waves transmission of BMFs from an in vivo externally placed body part (the human hand) received by a freshly tweezers plucked human hair. Still microphotographs and video recordings are presented documenting the effects of inductive BMFs, shown as an increase in crystals formation adjacent to the follicle and shaft attributed to an increase in energy reception of the

(C) 2020 The Author(s). This is an open access article distributed under the terms of the Creative Commons Attribution License, which permits unrestricted use, distribution, and reproduction in any medium, provided the original author and source are credited. 
inherent biomagnetic energy present in human hairs. Ferrocyanide crystallization was previously shown to be directly proportional to follicular energy levels [6].

\section{MATERIALS AND METHODS}

\subsection{MATERIALS}

- Potassium Ferrocyanide Trihydrate Glanular $\mathrm{K}_{4} \mathrm{Fe}(\mathrm{CN})_{6} .3 \mathrm{H}_{2} \mathrm{O}$.

- $\quad$ CSA \# 14459-95-1

- Microscope - Glass Slides: 25x75x1mm thickness. Pearl CAT N0. 7101

- Water purity confirmed by hand held electrical fields detector manufactured by Lishtot Detection LTD, Israel. For details link to: https://www.lishtot.com/TDP1.html

- $\quad$ Non-contact infrared thermometer Omega Model \# 0S425-LS

- $\quad$ Room relative humidity monitored by an ACU-RITE sensor model \# 01536-RX.

- Digital Video Microscope Celestron II model \# 44341, California, USA.

- Images downloaded to an Apple Computer MacBook Pro Photo Application.

\subsection{METHODS}

\subsubsection{PREPARING THE SOLUTION}

Commercially available bottled water was tested for impurities via a handheld electrical fields sensor. A solution was prepared by diluting $\cong 1$ gram of Potassium Ferrocyanide $\left(\mathrm{K} \mathrm{Fe}_{2}\right)$ in $1 \mathrm{ml}$ of the previously tested for impurities bottled spring water. The solution placed inside a 6" $4 \mathrm{~mm}$ OD glass tube and withdrawn via pipette.

\subsubsection{THE SINGLE SIDE PREPARATION (SSP)}

The SSP is an open-air technique where freshly plucked in toto scalp hairs were placed on a clean $25 \times 75 \times 1 \mathrm{~mm}$ glass slide; and covered by a drop of $\mathrm{KFe}_{2}$ in solution; the drop was which was allowed to evaporate. The drop was then touched by a wooden toothpick and dispersed as to cover the follicle and shaft (Figure 1).

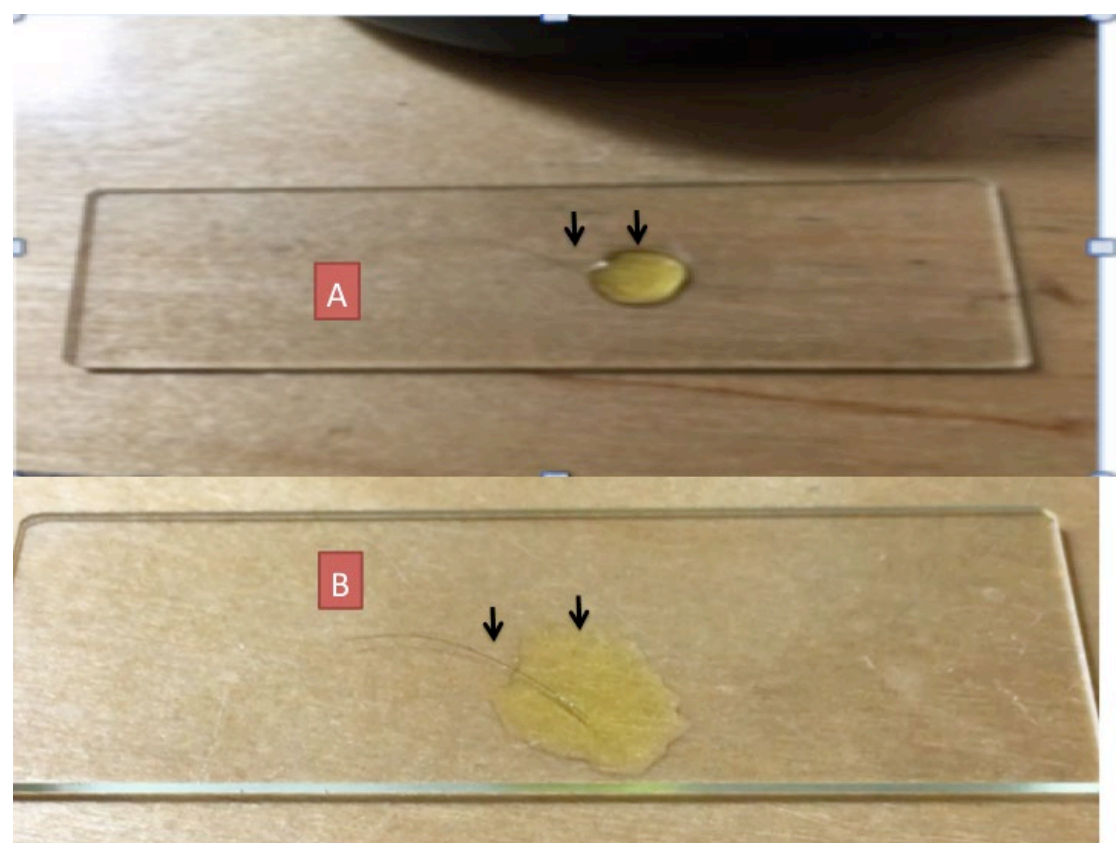

Figure 1: A: Scalp hair on glass slide covered by drop of KFe2 (Potassium Ferrocyanide) covering mainly the hair follicle. B: Same hair. Now the KFe2 drop surface tension disturbed via wooden toothpick now covering follicle and shaft. 
Demonstration of The Human Hair Follicle Magnetoreception Of Biomagnetism Radiated by The Concave Part of The Human Hand

\subsubsection{CONTROL SSP $\mathrm{KFE}_{2}$ EXPERIMENTS}

The SSP was allowed to unempidedly evaporate as shown in (Fig 2) below. Microphotographs and video recordings stored for future analysis,

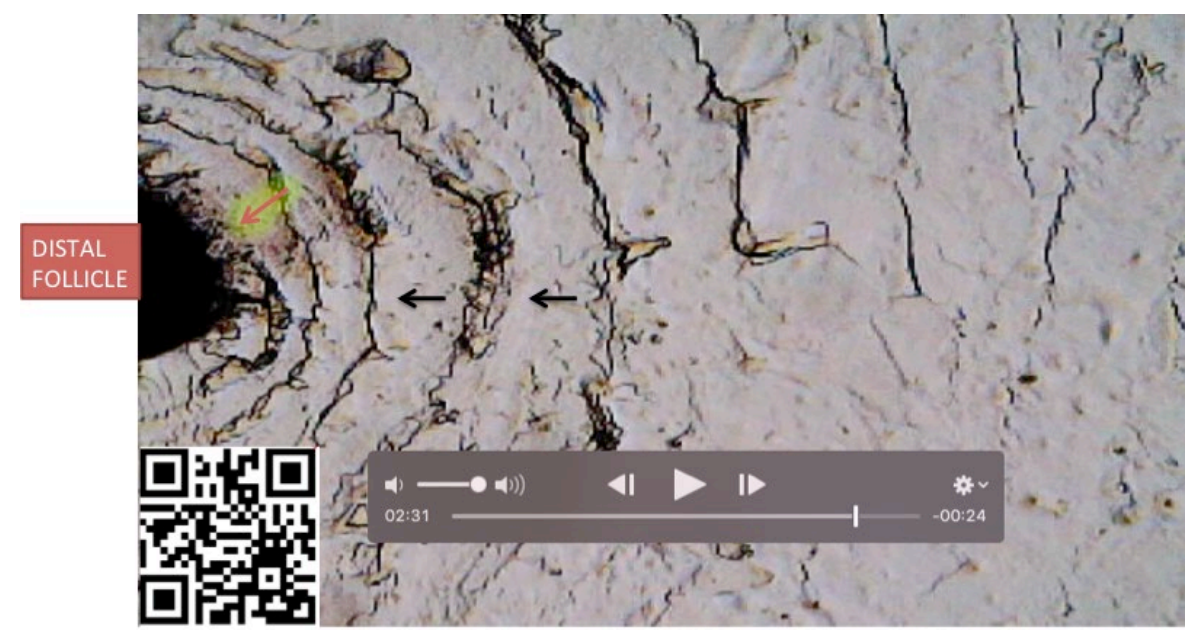

Figure 2: Control SSP $\mathrm{KFe}_{2}$ crystallization pattern when void of external BMFs influence- Black Arrows: Pointing at $\mathrm{KFe}_{2}$ crystals delineating the follicle diamagnetic property sequentially repelling the diamagnetic ferrocyanide. For details link to: https://youtu.be/eo6bYwJNlM0

Or scan QR Code in lower left corner of image.

\subsubsection{THE HAND PALM EXPERIMENTS}

The left hand was placed over (not touching) the preparation and held in place until the end of crystallization (approximate $7 \pm 3$ minutes). The room air temperature measured $81.3^{\circ} \mathrm{F}$, Relative Humidity $66 \%$, temperature under the palm increased one-degree F during the hand covering maneuver (Fig 4) below.

\subsubsection{ANCILLARY TEST TO RULE OUT TEMPERATURE AS CONTRIBUTING FACTOR}

A small inanimate wood stick fragment was placed on slide for a SSP $\mathrm{KFe}_{2}$ control experiment. As shown, there is a lack of crystallization accumulating or surrounding the distal part of the inanimate toothpick, thus demonstrating that BMFs inductive energy by the human hand is not the trigger for greater crystallization (Fig 3), in other words increased crystallization surrounding the hair follicle is directly proportional with an increase in metabolism (read energy).

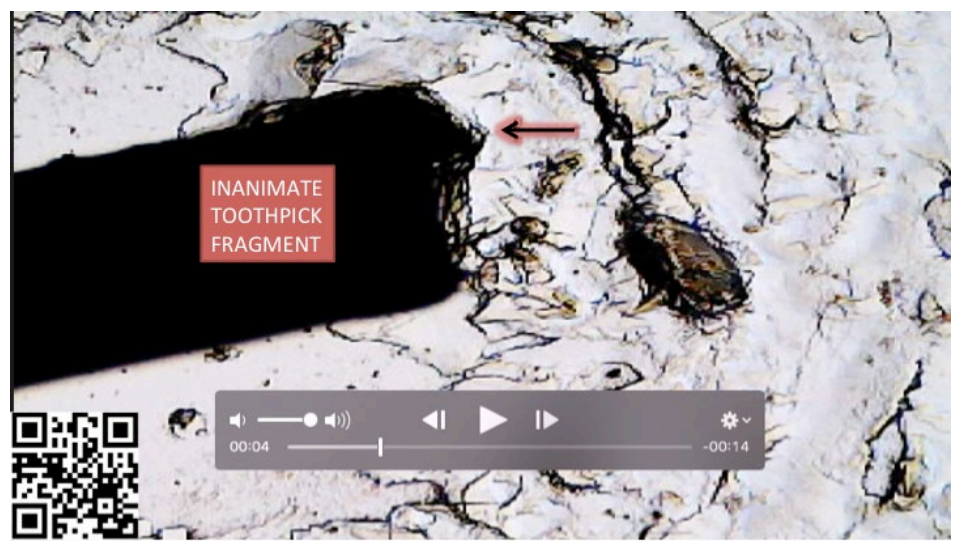

Figure 3: Supplemental experiment of hand covering of an inanimate object BMFs effect. Showing inanimate toothpick fragment in SSP $\mathrm{KFe}_{2}$ after evaporation. Black Arrow: Highlighting lack of crystallization surrounding fragment. Please compare with Figures 7 and 8 below. For details link to: https://youtu.be/8QYcZHQKn8A

Or Scan QR Code in left lower corner of image.

International Journal of Research -GRANTHAALAYAH 


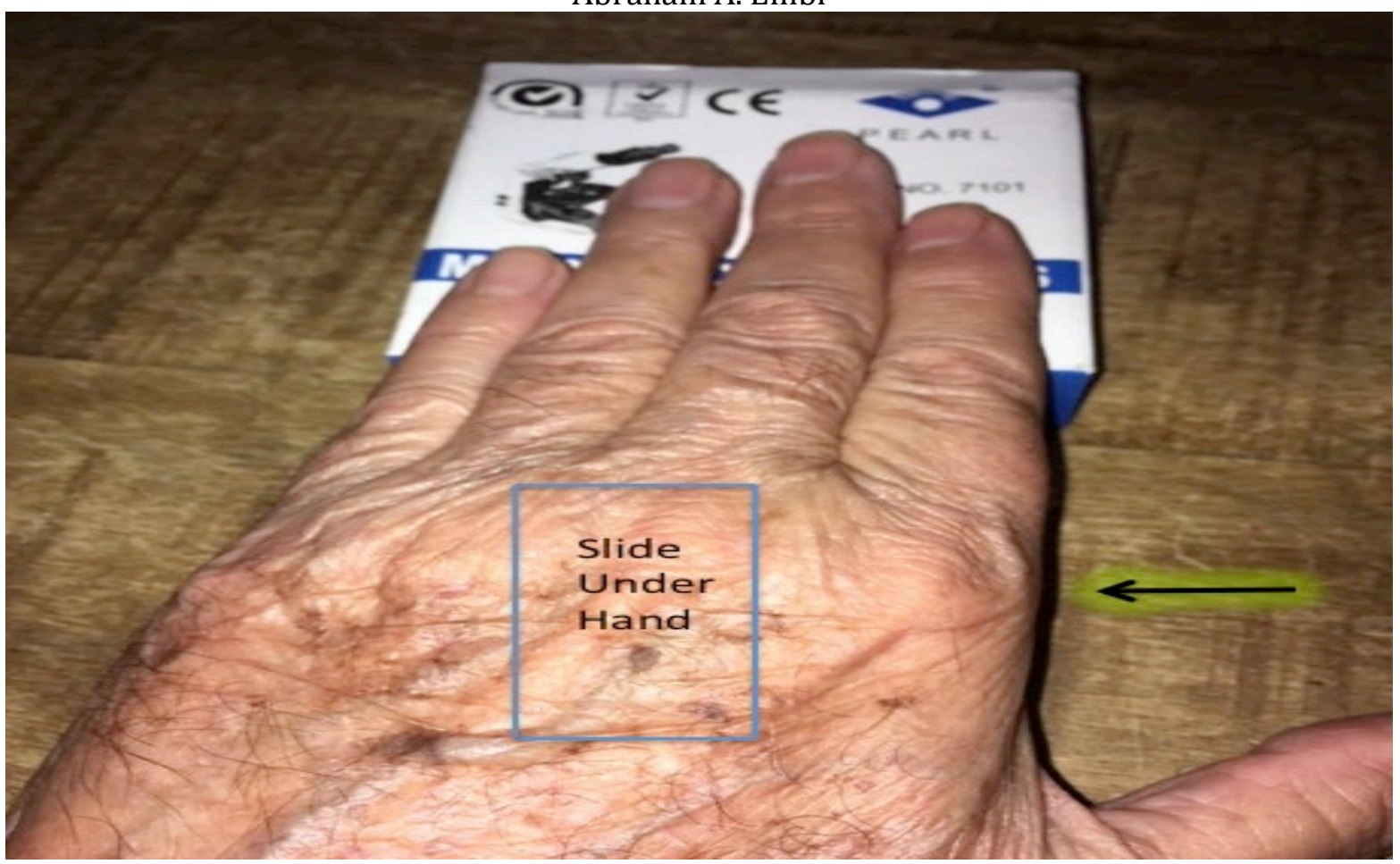

Figure 4: Left hand now indirectly covering at $\approx 1 \mathrm{~cm}$ vertical distance from the wet glass slide with Potassium Ferrocyanide (SSP $\mathrm{KFe}_{2}$ ) covering the plucked hair. Black Arrow: Pointing at area under the palm of the hand where slide was placed. There is no direct contact between the hand and the slide.

\section{RESULTS}

\subsection{DIAMAGNETIC POTASSIUM FERROCYANIDE}

Control experiments show a sequential crystallization waves surrounding the proximal hair follicle. This type of response has been documented and published in numerous previous papers; and can be appreciated in Figure 5 below:

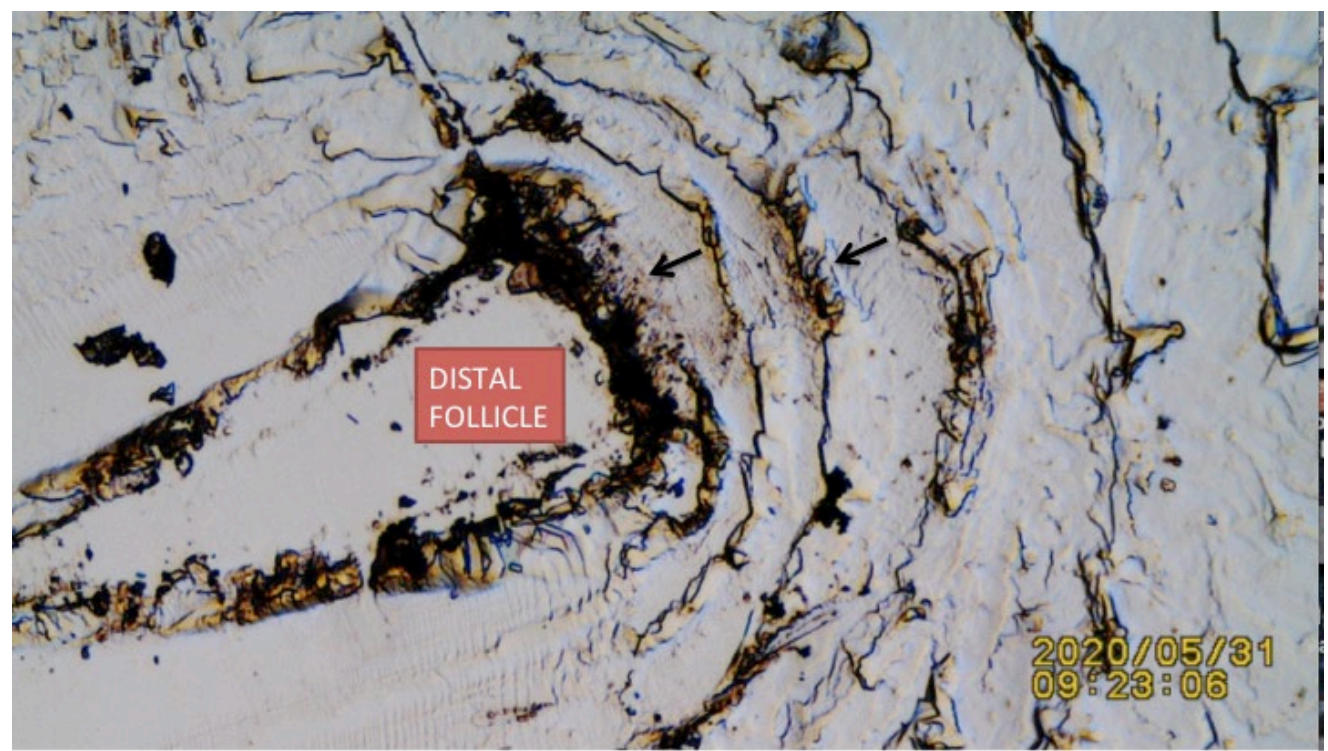

Figure 5: Same hair as in figure 2. Control hair in SSP $\mathrm{KFe}_{2}$. Image at end of crystallization and hair physically removed via toothpick, showing follicle imprint and "normal" crystallization pattern resulting from follicular BMFs. 
Demonstration of The Human Hair Follicle Magnetoreception Of Biomagnetism Radiated by The Concave Part of The Human Hand

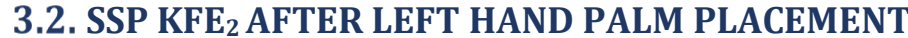

Opposite to the sequential crystallization patterns observed in the control experiments (Figures 2,3 above); there is now a stark difference in both the pattern and clumping of $\mathrm{KFe}_{2}$ crystals surrounding the hair follicle. This phenomenon is appreciated in Figures $(6,7,8)$ below.

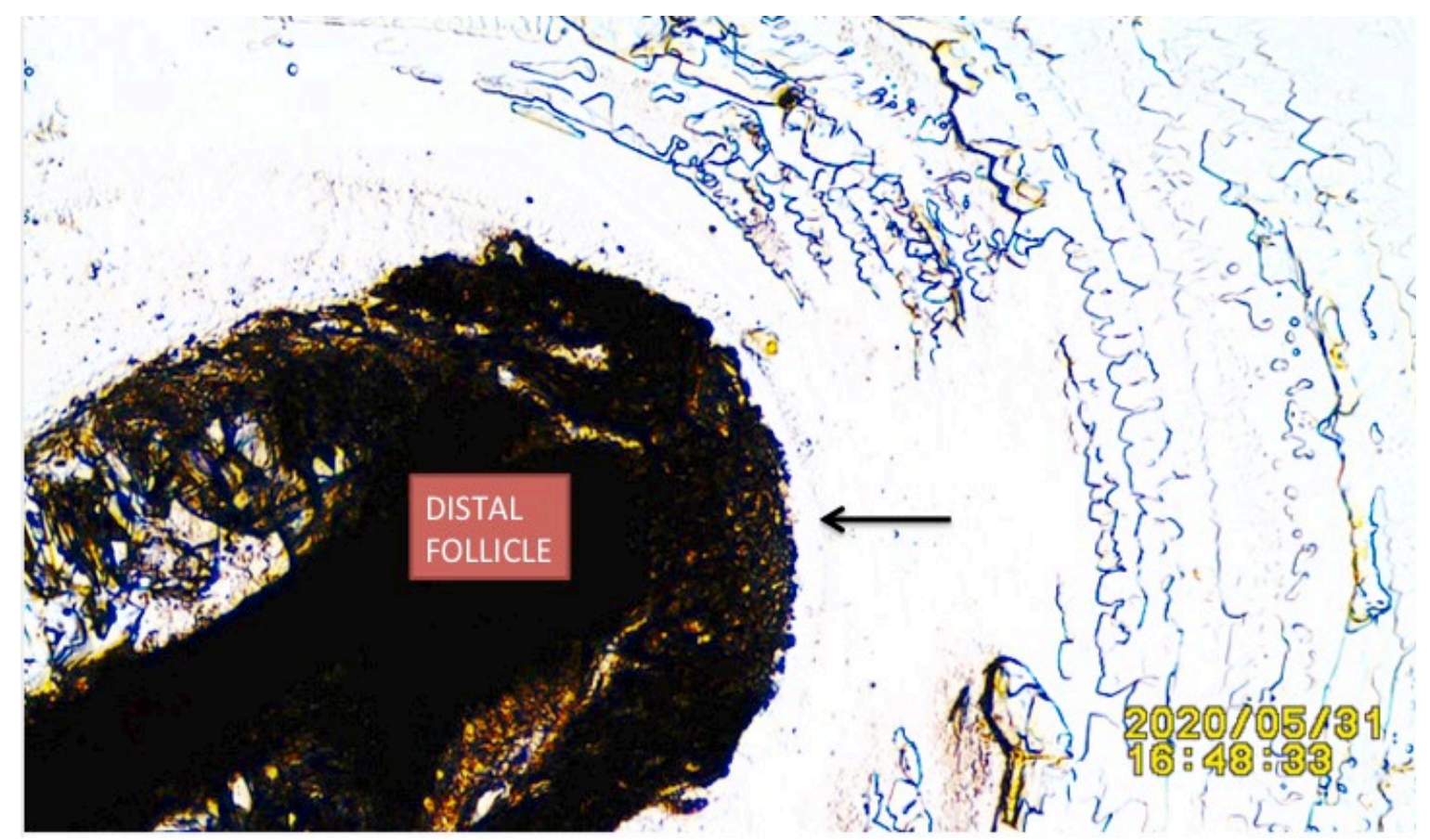

Figure 6

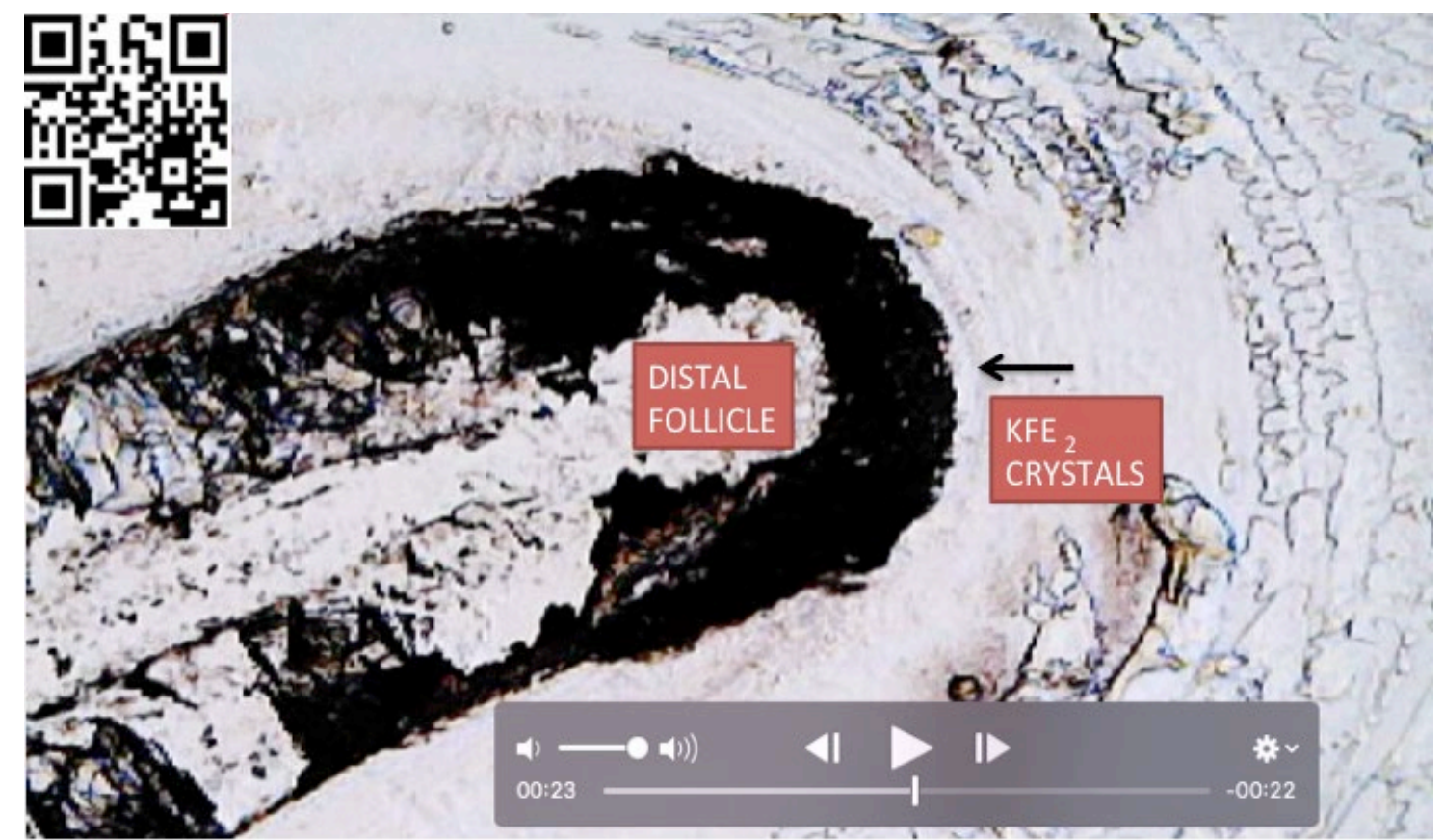

Figure 7: Hair follicle in SSP $\mathrm{KFe}_{2}$ influenced by BMFs from exposure to the indirect hand palm placement throughout the evaporation process. Hair proper removed via toothpick to show increased in crystallization.

Black Arrow: Notice stark increase in crystallization surrounding the distal hair follicle as compared with control (Figs 2,4 above). 


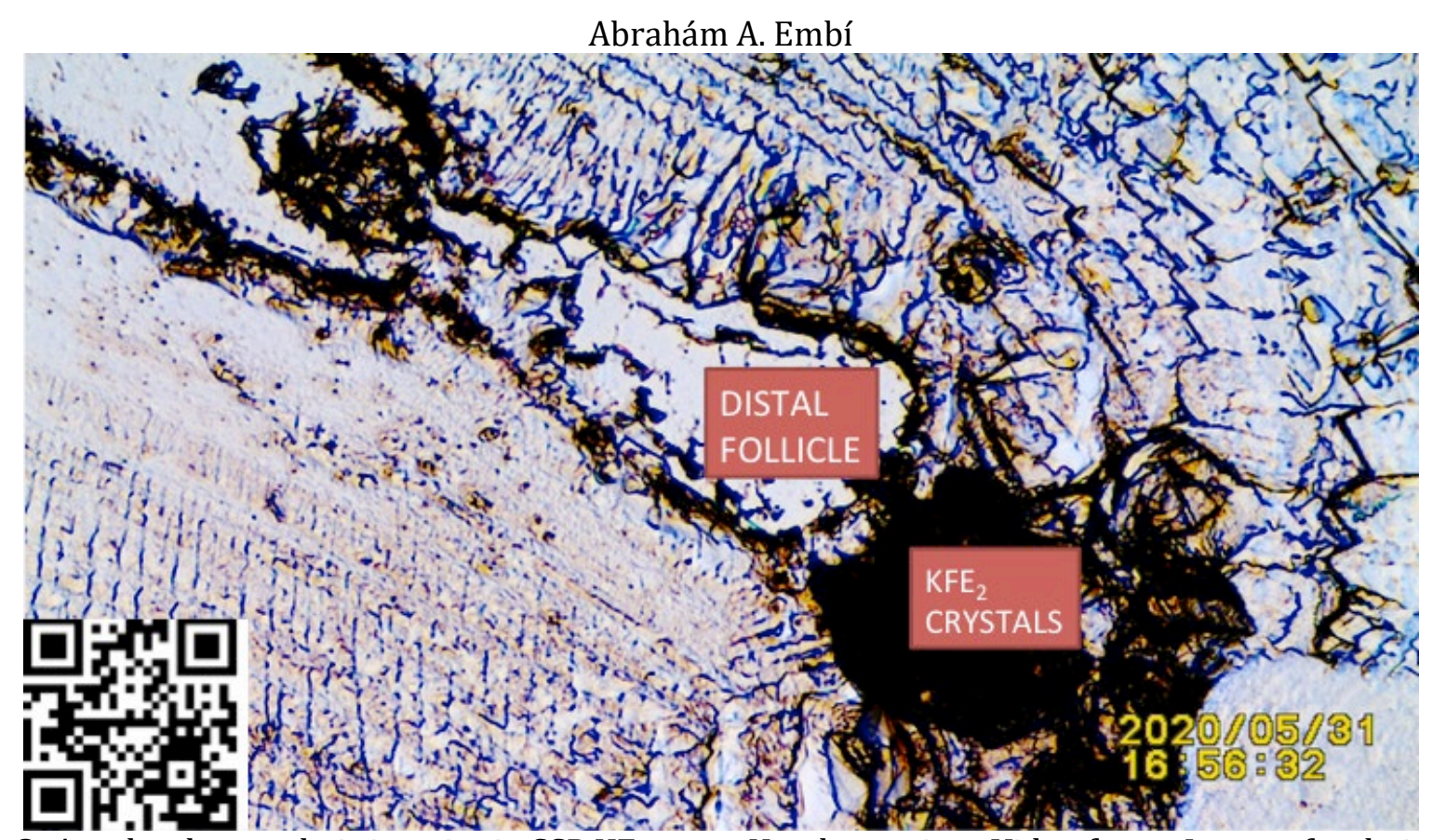

Figure 8: Another human hair imprint in SSP $\mathrm{KFe}_{2}$ post Hand covering. Video frame Image after hair proper removed to appreciate increased crystallization adhering to follicle.

For additional details link to https://youtu.be/OBQa3mEXYiE

Or Scan with QR Code in lower left side of image.

\section{DISCUSSION}

Biophysics is the interdisciplinary science that applies physical principles to study biological phenomena; and it has been also described as "The Bridging Science". Biomagnetism is the branch of Biophysics where magnetic signals are recorded arising from living entities and defined as "the generation of magnetic fields by living organisms. The hair follicle inherent electromagnetic energy had been documented, first by Cohen et al. in 1980 and subsequently by other researchers. Of interest to this manuscript is the property of hair emitted BMFs to penetrate glass barriers. This finding was possible due to an observation linking a trigger in crystallization of $\mathrm{KFe}_{2}$ in solution within the follicle's BMFs reach [7].

\subsection{INCREASED CRYSTALLIZATION OF KFE $\mathrm{BY}_{2}$ INCREASED METABOLISM}

The observed stark increase in crystallization surrounding the hair follicle is attributed to the aforementioned phenomenon of linking crystallization with biomagnetic energy transfer into the hair follicle. It supports BMFs energy transfer from a human body part to another.

\section{SOURCES OF FUNDING}

None.

\section{CONFLICT OF INTEREST}

None.

\section{ACKNOWLEDGMENT}

None. 
Demonstration of The Human Hair Follicle Magnetoreception Of Biomagnetism Radiated by The Concave Part REFERENCES

of The Human Hand

[1] Cohen D, Palti Y, Cuffin BN, Schmid SJ. (1980). Magnetic fields produced by steady currents in the body. Proc. Natl. Acad. Sci. USA; 77(3): 1447-1451.

[2] Khan, S., Cohen, D. (2019) Magnetotrichography: Measuring the dc magnetic field produced by hair follicles. Sci Rep 9, 15624. https://doi.org/10.1038/s41598-019-52110-y

[3] Benjamin J. Scherlag, Kaustuv Sahoo, Abraham A. Embi A. (2016) Novel and Simplified Method for Imaging the Electromagnetic Energy in Plant and Animal Tissues. Journal of Nanoscience and Nanoengineering Vol. 2, No. 1, pp.6-9

[4] Embi AA, Jacobson JI, Sahoo K, Scherlag BJ (2015) Demonstration of Inherent Electromagnetic Energy Emanating from Isolated Human Hairs. Journal of Nature and Science, 1(3): e55.

[5] Abraham A. Embi, Benjamin J. Scherlag. (2016) Demonstration of Human Hair Follicle Biomagnetic Penetration Through Glass Barriers. International Journal of Materials Chemistry and Physics Vol. 2, No. 2, pp. 71-74

[6] Embi AA, Scherlag BJ. (2015) Human Hair Follicle Biomagnetism: Potential Biochemical Correlates. J Mol Biochem; 4:32-35.

[7] Abraham A. Embi Bs. (2018). "THE HUMAN HAIR FOLLICLE PULSATING BIOMAGNETIC FIELD REACH AS MEASURED BY CRYSTALS ACCRETION." International Journal of Research - Granthaalayah, 6(7), 290-299. https://doi.org/10.5281/zenodo.1341349. 